\title{
Influenza study of backyard animals in Georgia
}

\author{
Anna Machablishvili¹, Lela Ursuhadze ${ }^{1}$ and Ivane Daraselia ${ }^{2}$ \\ ${ }^{1}$ National Center for Disease Control and Public Health, Tbilisi, Georgia; ${ }^{2}$ Tbilisi Zoological Park, Tbilisi, Georgia
}

\section{Objective}

The purpose of this study was to identify zoonotic influenza viruses in swine and poultry populations in Georgia and to define their pandemic potential.

\section{Introduction}

Aquatic birds are the main reservoirs of influenza viruses, however pigs represent an essential host in virus ecology as they are susceptible to both avian and human influenza viruses. Circulating zoonotic influenza (A/H7N9, A/H5N1, and A/H3N2v) viruses could mutate into forms easily transmissible from human-to-human and become a public health concern. Georgia is located along routes used by migrating birds where different species of aquatic birds are found. In 2006, highly pathogenic influenza virus A/H5N1 was detected in two wild swans in Adjara (western Georgia). Moreover, in the frame of wild bird surveillance, various subtypes of influenza A viruses were detected in mallard and gulls in Georgia (Lewis, 2013). Thus domestic animals in Georgia have a potential chance to contract influenza viruses from wild birds.

\section{Methods}

The Kakheti region, the leading region in cattle breeding and poultry production in Georgia, was selected for study. Villages were selected for door-to-door visits to search for ill backyard animals showing influenza-like symptoms. In case of identification of a sick animal, samples were obtained for laboratory investigations; sample collection forms were filled out to generate epidemiological data. Cloacal and tracheal swabs were taken from poultry; and pharyngeal and nasal swabs were collected from pigs. Each specimen was screened for influenza A matrix gene by real-time RT-PCR using a protocol from the Centers for Disease Control Prevention.

\section{Results}

Eighty four villages in the Kakheti region were surveyed for domestic animals with influenza-like illness symptoms. In total, 164 specimens were collected from 112 backyard animals in 55 households (107 samples were from 55 poultry and 57 samples were from 57 pigs). All samples tested negative for Influenza A virus by real time RT-PCR. The questionnaire data revealed that the age range of both pigs and poultry varied from one month to two years; median and mode were both 1 year. Chickens and ducks primarily freely ranged in backyards (67\%), while half the number of pigs were kept in closed premises. Equally, $61 \%$ of pigs and poultry had contact with other pigs or poultry within the premises.

\section{Conclusions}

In spite of the negative findings, we cannot exclude the circulation of influenza viruses in domestic animals in Georgia. Especially, considering the fact that a domestic duck with influenza A/H10 virus was identified during veterinarian training in 2010 in Grigoleti (Black sea cost of Georgia) manifesting no clinical symptoms. Therefore, larger scale studies, including swabbing more backyard animals without any clinical symptoms are necessary to identify interspecies virus transmission in the country.

\section{Keywords}

Zoonotic influenza; Pigs; Poultry; Georgia

\section{Acknowledgments}

Funding to conduct this study was provided by CRDF Georgia mini grant \#60211. Participation in this conference was made possible by financial support provided by the US Defense Threat Reduction Agency. The findings, opinions and views expressed herein belong to the authors and do not reflect an official position of the Department of the Army, Department of Defense, or the US Government, or any other organization listed.

\section{References}

Lewis NS, et al. (2013). Avian Influenza Virus Surveillance in Wild Birds in Georgia: 2009-2011. PLoS ONE 8(3): e58534. doi:10.1371/ journal.pone. 0058534

*Anna Machablishvili

E-mail: a_machablishvili@hotmail.com 